\title{
Communication \\ Evaluation of the Binding Kinetics of RHEB with mTORC1 by In-Cell and In Vitro Assays
}

\author{
Raef Shams ${ }^{1,2}$, , Yoshihiro Ito ${ }^{1,3(1)}$ and Hideyuki Miyatake ${ }^{2,3, *}$ \\ 1 Emergent Bioengineering Materials Research Team, RIKEN Center for Emergent Matter Science, RIKEN, \\ Wako 351-0198, Saitama, Japan; raef.shams@riken.jp (R.S.); y-ito@riken.jp (Y.I.) \\ 2 Department of Life Science, Graduate School of Science and Engineering, Saitama University, \\ Saitama City 338-8570, Saitama, Japan \\ 3 Nano Medical Engineering Laboratory, RIKEN Cluster for Pioneering Research, RIKEN, \\ Wako 351-0198, Saitama, Japan \\ * Correspondence: miyatake@riken.jp; Tel.: +81-48-467-4979
}

Citation: Shams, R.; Ito, Y.; Miyatake, $\mathrm{H}$. Evaluation of the Binding Kinetics of RHEB with mTORC1 by In-Cell and In Vitro Assays. Int. J. Mol. Sci. 2021, 22, 8766. https://doi.org/ $10.3390 /$ ijms 22168766

Academic Editor: Sławomir Filipek

Received: 10 June 2021

Accepted: 13 August 2021

Published: 16 August 2021

Publisher's Note: MDPI stays neutral with regard to jurisdictional claims in published maps and institutional affiliations.

Copyright: (c) 2021 by the authors. Licensee MDPI, Basel, Switzerland. This article is an open access article distributed under the terms and conditions of the Creative Commons Attribution (CC BY) license (https:// creativecommons.org/licenses/by/ $4.0 /)$.

\begin{abstract}
The mammalian/mechanistic target of rapamycin complex 1 (mTORC1) is activated by the small G-protein, Ras homolog enriched in brain (RHEB-GTPase). On lysosome, RHEB activates mTORC1 by binding the domains of N-heat, M-heat, and the focal adhesion targeting (FAT) domain, which allosterically regulates ATP binding in the active site for further phosphorylation. The crucial role of RHEB in regulating growth and survival through mTORC1 makes it a targetable site for anti-cancer therapeutics. However, the binding kinetics of RHEB to mTORC1 is still unknown at the molecular level. Therefore, we studied the kinetics by in vitro and in-cell protein-protein interaction (PPI) assays. To this end, we used the split-luciferase system (NanoBiT ${ }^{\circledR}$ ) for in-cell studies and prepared proteins for the in vitro measurements. Consequently, we demonstrated that RHEB binds to the whole mTOR both in the presence or absence of GTP $\gamma \mathrm{S}$, with five-fold weaker affinity in the presence of GTP $\gamma \mathrm{S}$. In addition, RHEB bound to the truncated mTOR fragments of N-heat domain $(\Delta N$, aa $60-167)$ or M-heat domain $(\Delta M$, aa 967-1023) with the same affinity in the absence of GTP. The reconstructed binding site of RHEB, $\triangle \mathrm{N}$-FAT-M, however, bound to RHEB with the same affinity as $\triangle \mathrm{N}-\mathrm{M}$, indicating that the FAT domain ( $\triangle \mathrm{FAT}$, aa 1240-1360) is dispensable for RHEB binding. Furthermore, RHEB bound to the truncated kinase domain ( $\triangle \mathrm{ATP}$, aa 2148-2300) with higher affinity than to $\triangle$ N-FAT-M. In conclusion, RHEB engages two different binding sites of mTOR, $\triangle$ N-FAT-M and $\triangle \mathrm{ATP}$, with higher affinity for $\triangle \mathrm{ATP}$, which likely regulates the kinase activity of mTOR through multiple different biding modes.
\end{abstract}

Keywords: mTORC1; RHEB; G-Protein; allosteric activation; kinase domain; binding kinetics

\section{Introduction}

The mammalian/mechanistic target of rapamycin (mTOR) regulates cell growth and survival through the modulation of the metabolic pathways [1,2]. mTOR assembles in two different complexes, mTOR complex 1 (mTORC1) and mTOR complex 2 (mTORC2), to regulate different processes $[1,3]$. In the mTORC1, mTOR is a kinase complexed with other proteins, regulatory associated protein of mTOR (Raptor), the mammalian lethal with SEC13 protein 8 (mLST8), DEP domain-containing mTOR-interacting protein (DEPTOR), and the 40-kDa proline-rich AKT substrate (PRAS40), which regulates the recruitment and phosphorylation of substrates (Figure 1a) [3,4]. In response to growth factors and nutrients, mTORC1 regulates a variety of life phenomena; synthesis of proteins, lipids and nucleotides, cell proliferation, and autophagy [1]. Recently, several studies have revealed the molecular mechanisms of mTORC1 kinase activation by amino acids and growth factors [5-7].

Early biochemical studies suggested that the small G-protein, Ras homolog enriched in brain (RHEB) was involved in the activation of mTORC1 [8-10]. In addition, it was 
found that the tuberous sclerosis complex $1 / 2$ (TSC1/2), the upstream negative regulator of mTORC1, served as a GTPase-activating protein for RHEB $[7,11,12]$. Through this, the active RHEB-GTPase positively modulates the mTORC1 activity. Later, it was shown that RHEB binds to the ATP binding domain (aa 2148-2300) in a GTP-independent manner [10], which does not activate the kinase activity. Therefore, the functional aspect of the binding still has to be addressed [10].

a

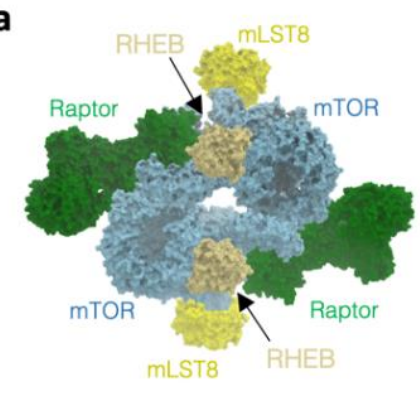

b

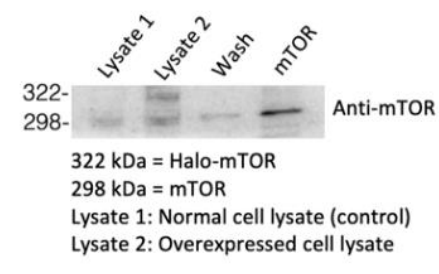

C

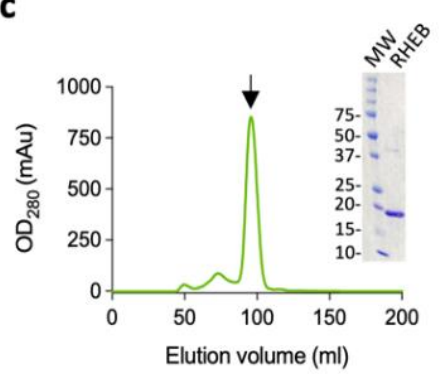

d

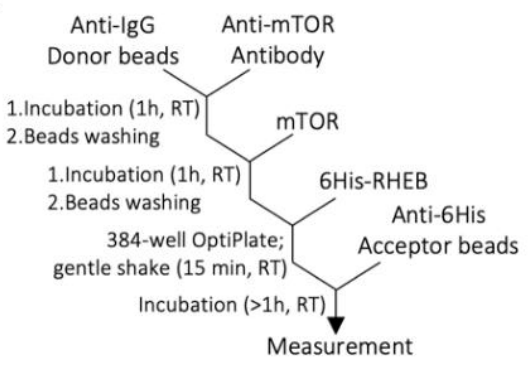

e

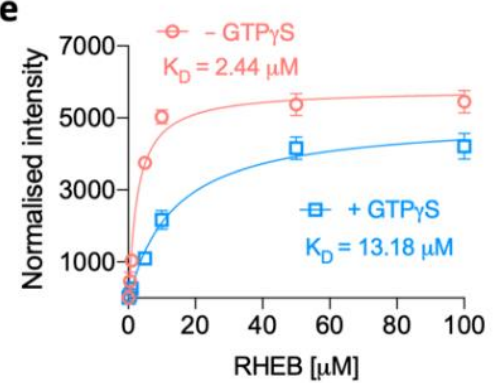

Figure 1. Binding kinetics of RHEB-mTOR. (a) Molecular structure of homodimeric mTORC1 showing the complex components except DEPTOR and PRAS40 (PDB ID: 6BCU) solved by the cryo-EM. (b) Western blot analysis of the different stages of mTOR purification showing the overexpression of the Halo-tagged mTOR. See the whole view of the blot in Figure S2b. (c) Gel-filtration profile and the corresponding Coomassie brilliant blue R-250 stained SDS-PAGE (cropped from the full gel image in Figure S3d) of the purified 6xHis-RHEB ( $M_{\mathrm{W}}$ : Molecular Weight; $\left.\mathrm{kDa}\right)$. The arrow indicates the elution peak to be analyzed. The weak band above $37 \mathrm{kDa}$ marker corresponds to the protein dimer. (d) Protocol of AlphaLISA ${ }^{\circledR}$ assay to measure the binding affinity of RHEB for mTOR. Different concentrations of $6 x H i s-R H E B$ and excess amount of anti-6His acceptor beads were used (see Figure S4 for details). RT, room temperature. (e) Binding of RHEB to mTOR in the presence (blue plot) or absence (red plot) of GTP $\gamma$ S. Data are shown as mean of two independent experiments $(n=3$ replicates each) \pm standard deviation (SD). The signal was normalized to the baseline. The equilibrium dissociation constants $\left(\mathrm{K}_{\mathrm{D}}\right)$ are shown.

It was reported that RHEB activates $\mathrm{mTORC} 1$ by antagonizing a negative regulator FKBP38, a member of FK506-binding protein family, in a GTP-dependent manner [8]. On the other hand, growth factors and nutrients promote the binding of the RHEB to mTOR, thereby promoting the kinase activity of mTORC1 [8]. Recently, the cryo-EM (cryogenic electron microscopy) structure of mTORC1/RHEB-GTP $\gamma \mathrm{S}$ complex showed the mechanism of the mTORC1 activation by RHEB-GTP [4]. In the complex, RHEBGTP $\gamma S$ bound to a binding site constituted by the N-heat, FAT, and M-heat domains far from the ATP binding site. It caused a large conformational change of mTOR, which in turn allosterically rearranged the ATP binding site to turn on the kinase activity [4]. Since RHEB is anchored to the lysosomal membrane mediated by farnesylation [12], the mTORC1 activation process occurs on the lysosome surface in response to growth factor and nutrient stimulation through two parallel and integrated pathways (Figure S1). At first, the RHEB-GTPase is being activated through the growth factor/TSC pathway, which enables RHEB to be charged by GTP [7,12]. Then, mTORC1 translocates onto the lysosome surface in response to the increasing concentration of amino acids $[5,6]$. The Raptor 
subunit of mTORC1 is anchored by the Rag GTPase-Ragulator complex onto the lysosome surface, offering the binding scaffold for RHEB-GTP $[13,14]$. On the lysosome surface, two RHEB-GTP complexes cooperatively activate mTORC1 to phosphorylate 4E-BP1 [4]. The stoichiometry obtained, however, did not involve the kinetics of RHEB binding to mTOR by means of protein-protein interaction (PPI) assays $[15,16]$. Therefore, in this study we aimed to reveal the kinetics of RHEB to mTOR, which can inform the development of new anti-cancer drugs.

\section{Results and Discussion}

Because mTORC1 and mTORC2 are often hyperactivated in cancer cells to sustain their rapid growth, its inhibition has been proposed for cancer therapy. Therefore, a variety of molecules have been developed to target the kinase activity of mTOR as anti-cancer agents [1,17-19]. However, specific inhibition of mTORC1 turned out to be more promising for cancer suppression than that of mTOR in both complexes. Thus, scientists have been seeking the ways to specifically target mTORC1 or, as a new strategy, to block signal transductions between mTORC1 and its regulatory proteins [11,20]. Accordingly, RHEB represents one of the potential targets for the specific inactivation of mTORC1 achieved by inhibiting the interaction between RHEB and mTORC1 [11]. Therefore, in this study, we aim to evaluate the binding kinetics of RHEB with mTOR to guide the development of new anti-cancer drugs.

At first, we studied the in vitro binding kinetics of RHEB to the whole mTOR. Briefly, the Halo-tagged full-length mTOR (aa 1-2,549; Kazusa-Promega, Supplementary Note 1 [4]) was overexpressed by the pFN21A/HEK293 cell system, and purified by the HaloLink ${ }^{\mathrm{TM}}$ resin (Promega, Madison, WI, USA) (Figure 1b and Figure S2). The artificial gene of RHEB was synthesized (Eurofins Genomics, Tokyo, Japan) and subcloned into pET15b expression vector (Novagen, Merck Millipore, Darmstadt, Germany). 6xHis-tagged RHEB (aa 1-169, Supplementary Note 2 [11]) was overexpressed in BL21(DE3) E. coli (Nippon gene, Toyama, Japan) and purified by Ni-NTA and Superdex-200 columns (GE Healthcare, Chicago, IL, USA) which was expressed as monomer/dimer mixture (Figure 1c and Figure S3). Next, we established the in vitro method for PPI determination by the AlphaLISA system including the anti-IgG donor beads and anti-6xHis acceptor beads (PerkinElmer, Waltham, MA, USA; Figure 1d and Figure S4). As a result, we observed that RHEB bound to mTOR in the presence or absence of GTP $\gamma \mathrm{S}$ (Merck Millipore, Darmstadt, Germany), although only RHEB-GTP was shown to activate mTORC1 [4]. However, the binding affinity of RHEB-GTP $\gamma \mathrm{S}$ to mTOR was five-fold weaker $\left(\mathrm{K}_{\mathrm{D}}=13.18 \mu \mathrm{M}\right)$ than that of GTP $\gamma$ S-free RHEB $\left(K_{D}=2.44 \mu \mathrm{M}\right.$; Figure 1e). This result suggests that a conformational change occurs upon GTP binding to RHEB, leading to the decreased binding affinity to mTOR. Because the GTP binding site is near the switch I of RHEB (aa 33-41), the binding of GTP probably interferes with the interaction between the switch I and mTOR domains involving M-heat and FAT [4].

The cryo-EM analysis revealed that RHEB interacted with three different mTOR fragments of aa $60-167$ in N-heat domain $(\Delta \mathrm{N})$, aa 967-1023 in M-heat domain $(\Delta \mathrm{M})$, and aa 1240-1360 in FAT domain ( $\triangle \mathrm{FAT}$ ) (Figure 2a) [4]. On the other hand, RHEB is also reported to bind the fragment aa $2148-2300$ of the ATP binding domain $(\triangle \mathrm{ATP})$, which is far from the RHEB binding site involving N-heat, M-heat, and FAT domains (Figure 2a) [10]. To reveal the binding properties of the mTOR fragments, we assayed the binding of RHEB with $\Delta \mathrm{N}, \Delta \mathrm{M}, \Delta \mathrm{N}-\mathrm{M}(\Delta \mathrm{N}+\Delta \mathrm{M}$ conjugates), $\Delta \mathrm{N}-\mathrm{FAT}-\mathrm{M}$ (constructed to mimic the $3 \mathrm{D}$ arrangement of the fragments in the RHEB binding site where the FAT domain combines the $\mathrm{N}$-heat and $\mathrm{M}$-heat domains to organize the allosteric binding site [4]) or $\triangle$ ATP by the split-luciferase technology (NanoBiT ${ }^{\circledR}$; Promega, Madison, WI, USA) (Figure S5) [21]. In the assay, the plasmids of RHEB-LgBiT and mTOR-SmBiT (representing different mTOR fragments) were co-transfected to HEK293 cells and incubated for $48 \mathrm{~h}$. Then, the PPI determinants were assayed by measuring the luminescence intensity initiated by the addition of furimazine (Nano-Glo, Promega, Madison, WI, USA; Figure 2b). As a 
result, RHEB bound to all the mTOR fragments with different affinities irrespective to the endogenous GTP levels. The luminescence intensity of $\triangle \mathrm{N}-\mathrm{M}$ and $\triangle \mathrm{N}$-FAT-M fell into the same range, suggesting that $\triangle F A T$ was little involved in RHEB binding (Figure 2c). The single fragments of $\Delta \mathrm{N}$ and $\Delta \mathrm{M}$ showed a similar luminescence intensity, suggesting the same level of affinity for RHEB (Figure 2c). In the bindings, the mTOR fragment conjugates of $\Delta \mathrm{N}-\mathrm{M}$ or $\Delta \mathrm{N}$-FAT-M showed higher affinity than those of $\Delta \mathrm{N}$ and $\Delta \mathrm{M}$, which suggested that the multiple fragments could increase the binding affinity in a cooperative manner (Figure 2c). In addition, $\triangle \mathrm{ATP}$ showed the highest luminescence intensity, suggesting the strongest affinity for RHEB (Figure 2c). This result corresponds with the previous report that RHEB interacted with the $\triangle$ ATP domain [10]. Since the $\triangle$ ATP domain of mTOR (aa 2148-2300) is highly conserved in the PI3K family [10,22], it is possible that RHEB regulates the kinase activity of mTOR upon the binding to $\triangle \mathrm{ATP}$ domain.

a

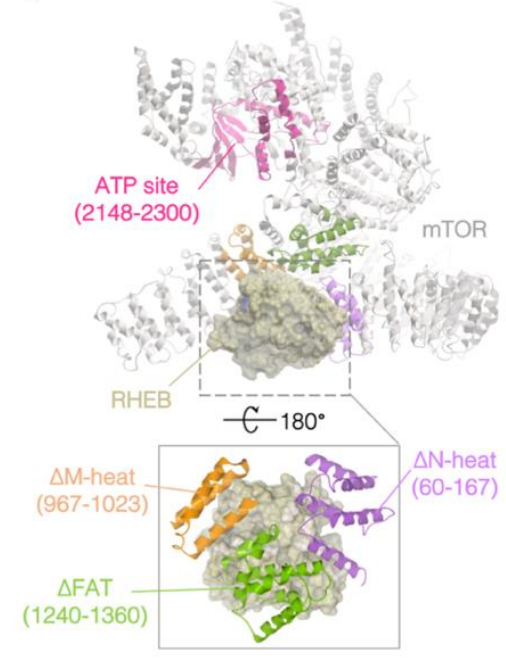

b

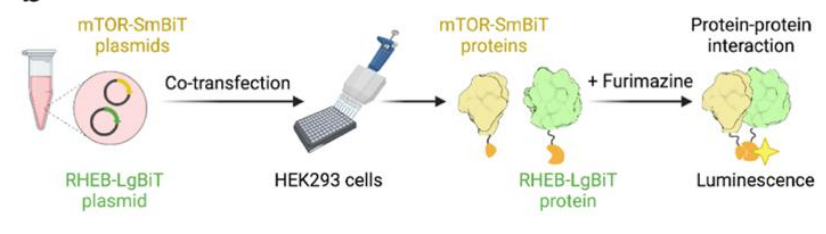

C

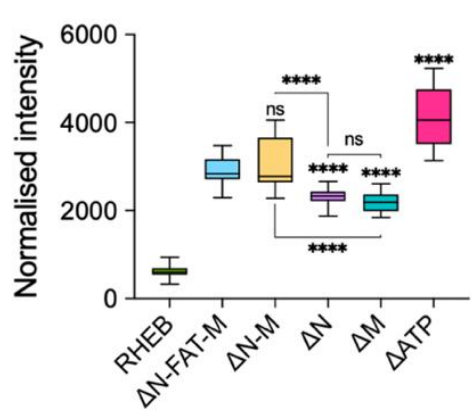

Figure 2. NanoBiT assay of RHEB binding to the different mTOR fragments. (a) The cryo-EM structure of RHEB complexed with mTOR (PDB ID: 6BCU) with the indicated mTOR fragments. $(\mathbf{b}, \mathbf{c})$ In-cell NanoBiT assay to evaluate the binding of the RHEB-LgBiT with the SmBiT-plasmids of the different mTOR fragments. (b) The plasmids were co-transfected to HEK293 cells, incubated for $48 \mathrm{~h}$, and the luminescence reaction was initiated by furimazine addition (created by Biorender.com (accessed on 1 June 2021)). (c) Luminescence of the mTOR fragments with RHEB. Data are shown as mean of two independent experiments $(n=6$ replicates each) \pm SD. The signal was normalized to the background. Ordinary one-way ANOVA was used: ${ }^{* * *} p<0.0001 ; \mathrm{ns}, p>0.05$.

Based on the NanoBiT results, we further quantitatively measured the binding kinetics of RHEB with the mTOR fragments of $\triangle \mathrm{N}-\mathrm{FAT}-\mathrm{M}, \Delta \mathrm{N}$, and $\triangle \mathrm{ATP}$ by the BLItz system (FortéBio, Fremont, CA, USA) [15]. For the measurements, we overexpressed the mTOR fragments by pET15b/BL21(DE3) E. coli system and purified them by Ni-NTA and Superdex-200 columns (GE Healthcare, USA) (Figures S6-S8). The 6xHis-tag was then cleaved by thrombin from RHEB and further purified by His SpinTrap column (GE Healthcare, USA) to remove the cleaved 6xHis-taggs, and then by benzamidine column (GE Healthcare, USA) to remove thrombin. After that, $1 \mu \mathrm{M}$ 6xHis-tagged mTOR fragments of $\triangle \mathrm{N}$-FAT-M, $\triangle \mathrm{N}$ or $\triangle \mathrm{ATP}$ were immobilized onto a Ni-NTA biosensor (FortéBio, Fremont, CA, USA) (Figure S9), and the 6xHis-tag cleaved-RHEB was used as analyte. As a result, RHEB interacted with the constructed allosteric binding site, $\triangle \mathrm{N}-\mathrm{FAT}-\mathrm{M}$, with $\mathrm{K}_{\mathrm{D}}=1.26 \mu \mathrm{M}$ (Figure $3 \mathrm{a}-\mathrm{c}$, Table 1 ). On the other hand, $\Delta \mathrm{N}$ showed a weaker affinity to RHEB with $\mathrm{K}_{\mathrm{D}}=6.47 \mu \mathrm{M}$ (Figure $3 \mathrm{~d}-\mathrm{f}$, Table 1), which corresponded to the in-cell results. Finally, RHEB bound to the $\triangle$ ATP with the highest affinity of $K_{D}=29 \mathrm{nM}$ (Figure $3 g-i$, Table 1), as suggested by the in-cell assay (Figure 2c). These different binding affinities suggest the multiple functionalities of RHEB to regulate the kinase activity of mTORC1 [4]. 
We tried to measure the binding kinetics of the whole mTOR by the same method, through the immobilization of RHEB onto the Ni-NTA biosensor and using mTOR as analyte, but we could not figure it out due to the fast association/dissociation rates owing to the large molecular weight of mTOR complexes.
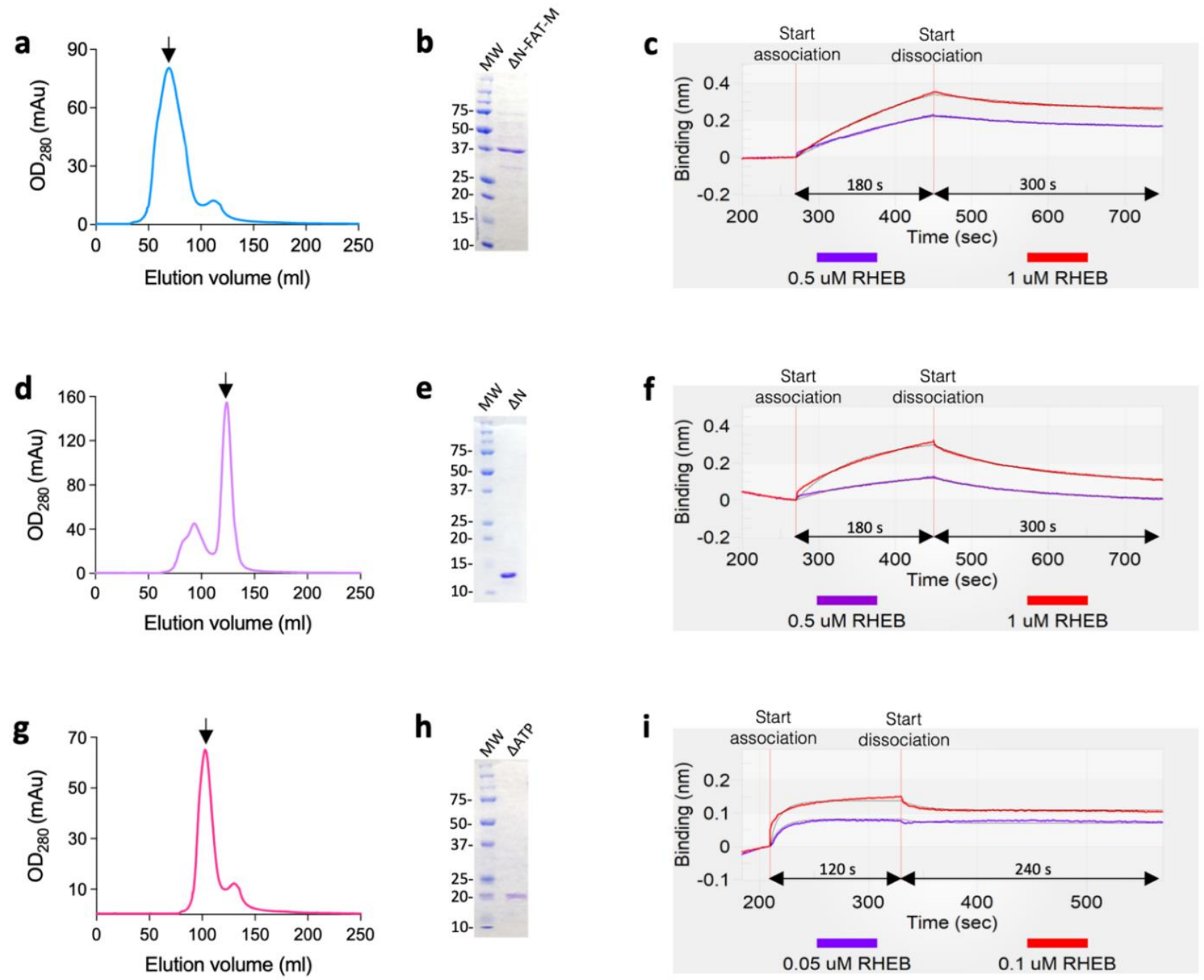

Figure 3. Binding kinetics of RHEB with mTOR fragments $(1 \mu \mathrm{M})$ measured by BLItz. (a,b) Gel filtration profile (a) and the corresponding Coomassie brilliant blue R-250 stained SDS-PAGE (b) of $\triangle$ N-FAT-M. See Figure S6d for full gel image. (c) Binding of RHEB to $\triangle$ N-FAT-M with association and dissociation phases of $180 \mathrm{~s}(270-450 \mathrm{~s})$ and 300 s (450-750 s), respectively. (d,e) Gel filtration profile (d) and a representative Coomassie brilliant blue R-250 stained SDS-PAGE (e) of $\Delta$ N. See Figure S7e for full gel image. (f) Binding of RHEB to $\Delta \mathrm{N}$ with association and dissociation phases of (270-450 s) and $300 \mathrm{~s}$ (450-750 s), respectively. (g,h) Gel filtration profile (g) and a representative Coomassie brilliant blue R-250 stained SDS-PAGE (h) of $\triangle$ ATP. See Figure S8e for full gel image. (i) Binding of RHEB to $\triangle$ ATP with association and dissociation phases of 120 s (210-330 s) and 240 s (330-570 s), respectively. Global fitting was carried out for 1:1 binding kinetics. The calculated parameters are shown in Table 1. The arrows indicate the elution peaks used for the analysis.

Table 1. Kinetic parameters of 1:1 binding model of RHEB with the indicated proteins.

\begin{tabular}{ccccc}
\hline Protein & $\mathbf{K}_{\mathbf{D}} \mathbf{( M )} \mathbf{1}^{\mathbf{1} *}$ & $\mathbf{k}_{\mathbf{a}}\left(\mathbf{M}^{-\mathbf{1}} \mathbf{s}^{-\mathbf{1}}\right)^{\mathbf{2} * *}$ & $\left.\mathbf{k}_{\mathbf{d}} \mathbf{s}^{-\mathbf{1}}\right)^{\mathbf{3} *}$ & $\chi^{\mathbf{2}}$ \\
\hline$\Delta$ N-FAT-M & $1.26 \pm 0.11 \times 10^{-6}$ & $2.00 \pm 0.12 \times 10^{3}$ & $2.40 \pm 0.11 \times 10^{-3}$ & 0.04 \\
$\Delta$ N & $6.47 \pm 0.13 \times 10^{-6}$ & $1.51 \pm 0.30 \times 10^{3}$ & $9.77 \pm 0.07 \times 10^{-3}$ & 0.04 \\
$\Delta$ ATP & $2.91 \pm 0.10 \times 10^{-8}$ & $8.50 \pm 0.13 \times 10^{5}$ & $2.47 \pm 0.03 \times 10^{-2}$ & 0.02 \\
\hline
\end{tabular}

\footnotetext{
${ }^{1} \mathrm{~K}_{\mathrm{D}}$, equilibrium dissociation constant. ${ }^{2} \mathrm{k}_{\mathrm{a}}$, association rate constant. ${ }^{3} \mathrm{k}_{\mathrm{d}}$, dissociation rate constant. ${ }^{4} \chi^{2}$, Chi-squared test of the fitted curve. ${ }^{*}$ The values of $\mathrm{K}_{\mathrm{D}}, \mathrm{k}_{\mathrm{a}}$, and $\mathrm{k}_{\mathrm{d}}$ are indicated \pm the standard errors.
} 
Overall, our study suggests that RHEB binds to mTORC1 in the presence or absence of GTP, suggesting multiple modes of RHEB to regulate mTORC1 activity. Previously, it has been reported that REHB activates mTORC1 in the presence of GTP $[4,9,10,23]$. In the activation, GTP changes the conformations of switch I and switch II regions of RHEB, respectively, to bind the RHEB binding site of mTORC1 [4]. In this study, however, we found that RHEB binds mTORC1 with higher affinity in the absence of GTP than that in the presence of GTP. We could resolve the inconsistency by assuming another binding site of mTORC1 for RHEB, even in the absence of GTP. The kinase domain of mTORC1 is reported to interact with RHEB $[9,10]$, which suggests that the domain is involved in the RHEB binding in the absence of GTP. To confirm this, we measured the binding affinities of RHEB for the truncated kinase domain $(\triangle \mathrm{ATP})$ and the fragmented allosteric binding site ( $\triangle$ N-FAT-M) in the absence of GTP, respectively. As a result, we found that RHEB binds to $\triangle$ ATP with much higher affinity than that of $\triangle \mathrm{N}$-FAT-M both in-cell and in vitro, which suggests that RHEB binds to the kinase domain of mTOR in the absence of GTP. Like the allosteric inhibition mode of rapamycin by binding to the kinase domain of mTORC1 with FKBP12 [24], RHEB may inhibit the kinase activity by posing a steric hindrance for the binding of ATP and/or the substrate proteins, providing a negative regulation mode for the kinase activity. Further studies should be conducted to confirm the scenario.

On the other hand, the FAT domain constructs the allosteric RHEB binding site, together with $\mathrm{N}$-heat and M-heat domains [4]. However, our study showed that RHEB binds $\triangle \mathrm{N}$-FAT-M and $\triangle \mathrm{N}$-M fragments with the same affinity, suggesting little contribution of FAT domain for the RHEB binding. This information will contribute to designing a variety of peptide-based anti-cancer drugs to inhibit the kinase activity of mTORC1 by interfering with the mTORC1-RHEB interaction. The targeting of RHEB by compounds is emerging as a new modality for cancer therapy [11]. Therefore, this study will inform us to develop new inhibitors for mTORC1, based on the kinetics of RHEB obtained.

\section{Materials and Methods}

\subsection{Materials}

All the materials and softwares used in this study were listed in Table 2.

\subsection{Methods}

\subsection{1. mTOR Expression and Purification}

Human mTOR ORF was supplied in pFN21A HaloTag ${ }^{\circledR}$ CMV Flexi ${ }^{\circledR}$ Vector (Kazusa Institute/Promega, Chiba, Japan), which was handled as previously described $[25,26]$. Briefly, HEK293 (RIKEN Cell Bank, RIKEN, Wako, Japan) cells were transfected by the Halo-tagged mTOR using FuGENE HD (Promega, Madison, WI, USA) transfection agent and grown as monolayer in $10 \mathrm{~cm}$ plates. Then, $10^{8}$ cells were collected and lysed on ice by $50 \mathrm{mM}$ HEPES, pH 7.5, $150 \mathrm{mM} \mathrm{NaCl}, 1 \mathrm{mM}$ EDTA, $1 \%(v / v)$ Triton X-100, 10\% (v/v) glycerol, $0.1 \%(w / v)$ sodium deoxycholate, $0.005 \%(v / v)$ IGEPAL CA-630 buffer containing protease inhibitor cocktail tablets (cOmplete ${ }^{\mathrm{TM}}$, Roche (Merck), Darmstadt, Germany), and $1.6 \mu \mathrm{g} / \mathrm{mL}$ DNase I. Cell lysate was then centrifuged at $13,200 \times \mathrm{g}$ for $30 \mathrm{~min}$ at $4{ }^{\circ} \mathrm{C}$, and the pellet was discarded. The supernatant was incubated with the pre-equilibrated HaloLink resin (Promega, Madison, WI, USA) for $1 \mathrm{~h}$ at RT on a rotator. Then, the resin was washed three times with the purification buffer. After that, mTOR was released from the resin by the TEV protease cleavage for further $1 \mathrm{~h}$ at RT on rotor. The released mTOR in the supernatant was carefully removed and incubated with Ni-NTA resin to remove the 6xHis-proteolytic TEV. Finally, the last supernatant containing mTOR was replaced by storage buffer (50 mM HEPES, $\mathrm{pH} 7.5,10 \%(v / v)$ glycerol) and concentrated by the $30 \mathrm{kDa}$ molecular weight cut-off (MW-CO) Amicon Ultra ${ }^{\circledR}$ (Millipore (Merck), Darmstadt, Germany) to be stored at $-80{ }^{\circ} \mathrm{C}$. The yield was examined by western blotting using anti-mTOR antibody. 
Table 2. Materials and softwares used in the study.

\begin{tabular}{|c|c|c|}
\hline Reagent or Resource & Source & Identifier \\
\hline \multicolumn{3}{|c|}{ Antibodies } \\
\hline Rabbit $\mathrm{mAb}$ anti-mTOR & Cell Signaling Technology & 2983; RRID: AB_2105622 \\
\hline Goat anti-rabbit-HRP secondary antibody & Invitrogen, Thermo Fisher & A16104; RRID: AB_2534776 \\
\hline \multicolumn{3}{|c|}{ Bacterial Strains } \\
\hline ECOS $^{\mathrm{TM}}$ Competent E. coli DH5 $\alpha$ & Nippon Gene & $316-06233$ \\
\hline ECOS $^{\mathrm{TM}}$ Competent E. coli BL21(DE3) & Nippon Gene & $312-06534$ \\
\hline \multicolumn{3}{|c|}{ Chemicals and Recombinant Proteins } \\
\hline Dpn1 enzyme & Takara & $1235 \mathrm{~A}$ \\
\hline ExoSAP-IT enzyme & Bioscience, Thermo Fisher & 75001.1 \\
\hline KOD one PCR enzyme mix & Toyobo & KMM-201 \\
\hline DMEM (High-Glucose) media & $\begin{array}{c}\text { FujiFilm Wako Pure } \\
\text { Chemicals }\end{array}$ & 044-29765 \\
\hline Opti-MEM media & Gibco, Thermo Fisher & $31985-070$ \\
\hline FuGENE HD & Promega & E2311 \\
\hline Ampicillin, sodium salt & Nacalai Tesque & 02739-32 \\
\hline Kanamycin & $\begin{array}{c}\text { FujiFilm Wako Pure } \\
\text { Chemicals }\end{array}$ & $113-00343$ \\
\hline Isopropyl $\beta$-D-1-thiogalactopyranoside (IPTG) & Nacalai Tesque & $19742-94$ \\
\hline IGEPAL CA-630 & MP Biomedicals & 198596 \\
\hline Luria-Bertani agar media & Sigma-Aldrich & 1002650948 \\
\hline Luria-Bertani Broth media & Nacalai Tesque & $20068-75$ \\
\hline Modified Terrific Broth media & Sigma-Aldrich & 1002891164 \\
\hline Antifoam SI & $\begin{array}{c}\text { FujiFilm Wako Pure } \\
\text { Chemicals }\end{array}$ & 018-17435 \\
\hline Protease inhibitors & Roche (Merck) & 06538282001 \\
\hline Thrombin & $\begin{array}{l}\text { FujiFilm Wako Pure } \\
\text { Chemicals }\end{array}$ & 206-18411 \\
\hline Coomassie brilliant blue R-250 & $\begin{array}{l}\text { FujiFilm Wako Pure } \\
\text { Chemicals }\end{array}$ & $6104-59-2$ \\
\hline $\mathrm{GTP}_{\gamma} \mathrm{S}$ & Millipore & $20-176$ \\
\hline \multicolumn{3}{|c|}{ Commercial kits } \\
\hline NucleoSpin ${ }^{\circledR}$ EasyPure kit & Macherey-Nagel & 740727.50 \\
\hline NanoBiT $^{\circledR}$ PPI Control Pair (FKBP/FRB) & Promega & N2016 \\
\hline Nano-Glo ${ }^{\circledR}$ Live Cell Assay System & Promega & N2012 \\
\hline Anti-Rabbit IgG Alpha Donor beads & PerkinElmer & AS105M \\
\hline Anti-6xHis AlphaLISA Acceptor beads & PerkinElmer & AL178M \\
\hline HaloTag protein purification system & Promega & G6270 \\
\hline In-Fusion cloning kit & Takara & 639650 \\
\hline HisTrap $^{\mathrm{TM}} \mathrm{HP}$ Ni column & Cytiva & 17524802 \\
\hline HisTrap $^{\mathrm{TM}}$ FF crude Ni column & Cytiva & 17528601 \\
\hline Superdex-200 HiLoad 16/60 column & GE Healthcare & 28-9893-35 \\
\hline His SpinTrap ${ }^{\mathrm{TM}}$ column & GE Healthcare & 28401353 \\
\hline HiTrap $^{\mathrm{TM}}$ Benzamidine FF column & GE Healthcare & $17-5143-02$ \\
\hline PD spintrap G-25 & GE Healthcare & 28918004 \\
\hline \multicolumn{3}{|c|}{ Cell Lines } \\
\hline HEK293 & RIKEN Cell Bank & $\mathrm{N} / \mathrm{A}$ \\
\hline
\end{tabular}


Table 2. Cont.

\begin{tabular}{ccc}
\hline Reagent or Resource & Source & Identifier \\
\hline $\begin{array}{c}\text { Synthetic human RHEB gene (507 bp) } \\
\text { UniportKB ID: Q15382 }\end{array}$ & Oligonucleotides & GSY1601-1 \\
\hline $\begin{array}{c}\text { Human mTOR ORF/pFN21A } \\
\text { UniportKB ID: P42345 }\end{array}$ & Kazusa Institute / Promega & FHC01207 \\
\hline pET15b vector & Novagen & 69661
\end{tabular}

Primers for RHEB fragmentation for $p E T 15 b$ :

$$
\text { FOR: }
$$

'GTGCCGCGCGGGCAGCCAGTCCAAAAGCCGCAAAATC'

REV: 'ATCGATAAGCTTCTATTCCAACTTTTCCGCTTCCAG'

Primers for $\mathrm{pET} 15 \mathrm{~b}$ linearization:

FOR: 'CATATGGCTGCCGCGCGGCACCAGGCCGCTGCTG'

REV: 'TAGAAGCTTATCGATGATAAGCTGTCAAACATGAG'

Primers for RHEB fragmentation for $\operatorname{LBBiT}$ :

$$
\text { FOR: }
$$

'ATCGCCATGGTGGCCCAGTCCAAAAGCCGCAAAATC'

REV: 'ACTGCCTTGAGAAACTTCCAACTTTTCCGCTTCC'

\section{Primers for LgBiT vector linearization:} FOR:

'GTTTCTCAAGGCAGTTCAGGTGGTGGCGGGAGCGG'

REV: 'GGCCACCATGGCGATCGCTAGCGGTGGCTTTACC'

\section{Primers for SmBiT vector linearization:}

$$
\text { FOR: }
$$

'TGGGCTAGCAGATCTTCTAGAGTCGGGGCGGCCGG'

REV: 'CATTCCACCGCTCGAGCCTCCACCTCCGCTCCCGC'

Primers for mTOR $^{\Delta N}$ Fragment for SmBiT:

FOR: 'GGCTCGAGCGGTGGATCTACTCGCTTCTATGACC'

REV: 'AGAAGATCTGCTAGCACCCAGCCATTCCAGGGC'

Primers for $\mathrm{mTOR}^{\Delta \mathrm{M}}$ Fragment for SmBiT:

FOR: 'GGCTCGAGCGGTGGACATCACACCATGGTTGTCC' REV:

'AGAAGATCTGCTAGCCACAAAGGACACCAACATTC'

Primers for $\mathrm{mTOR}^{\Delta \mathrm{N}-\mathrm{M}}$ Fragment for SmBiT:

FOR: 'ACATGCACATCACACCATGGTTGTCCAGGCCATC'

REV: 'GTGTGATGTGCATGTCTCCGGCCCTCATTGCGG'

Eurofins Genomics N/A
Eurofins Genomics
$\mathrm{N} / \mathrm{A}$

Eurofins Genomics N/A

Primers for mTOR ${ }^{\Delta \mathrm{N}-\mathrm{F}-\mathrm{M}}$ Fragment for SmBiT:

FOR: 'GGCCGGAGACATGCAGGCCAAGGGGATGCATTGG'

REV: 'AACCATGGTGTGATGCAAGTTTAAGAGGGTCTGTG'

Primers for mTOR ${ }^{\triangle A T P}$ Fragment for SmBiT:

FOR: 'GCTCGAGCGGTGGACAGCCAATCATTCGCATTCAG' REV:

Eurofins Genomics

$\mathrm{N} / \mathrm{A}$

Eurofins Genomics

$\mathrm{N} / \mathrm{A}$

Eurofins Genomics

$\mathrm{N} / \mathrm{A}$

Eurofins Genomics N/A

Eurofins Genomics N/A

Eurofins Genomics N/A

Eurofins Genomics N/A

'AGAAGATCTGCTAGCGGCCAGGTCGTCCCCAGCTG'

Primers for $\mathrm{mTOR}^{\Delta \mathrm{N}}$ Fragment for $\mathrm{pET} 15 \mathrm{~b}$ :

FOR: 'GTGCCGCGCGGCAGCTCTACTCGCTTCTATGACC'

REV: 'ATCGATAAGCTTCTAACCCAGCCATTCCAGGGCTC'

Eurofins Genomics

$\mathrm{N} / \mathrm{A}$

Primers for mTOR $^{\Delta N-F-M}$ Fragment for pET15b:

FOR: 'GATAACGCGATCGCCTCTACTCGCTTCTATGACC'

REV: 'CGAATTCGTTTAAACCACAAAGGACACCAACATTC'
N/A 
Table 2. Cont.

\begin{tabular}{|c|c|c|}
\hline Reagent or Resource & Source & Identifier \\
\hline $\begin{array}{c}\text { Primers for mTOR }{ }^{\Delta A T P} \text { Fragment for pET15b: } \\
\text { FOR: 'GATAACGCGATCGCCCTGCCTCAGCTCACATCC' } \\
\text { REV: 'CGAATTCGTTTAAACGCATGTGATTCTGTAGTTGC' }\end{array}$ & Eurofins Genomics & $\mathrm{N} / \mathrm{A}$ \\
\hline $\begin{array}{c}\text { Primers for colony PCR of SmBiT/LgBiT: } \\
\text { FOR: 'GAAGTCGAACACGCAGATGCAGTCG' } \\
\text { REV: 'CACTGCATTCTAGTTGTGGTTTGTCCAAACTC' }\end{array}$ & Eurofins Genomics & $\mathrm{N} / \mathrm{A}$ \\
\hline $\begin{array}{c}\text { Primers for colony PCR of pET15b: } \\
\text { FOR: 'CGATCCCGCGAAATTAATACGACTCACTATAG' } \\
\text { REV: 'GACATTAACCTATAAAAATAGGCGTATCACGAGG' }\end{array}$ & Eurofins Genomics & $\mathrm{N} / \mathrm{A}$ \\
\hline \multicolumn{3}{|c|}{ Software } \\
\hline ICM-Pro 3.9 software & Molsoft L.L.C. & $\begin{array}{c}\text { https://www.molsoft.com/ } \\
\text { products.html }\end{array}$ \\
\hline SnapGene 5.1 .7 software & GSL Biotech L.L.C. & $\begin{array}{c}\text { https: } \\
\text { //www.snapgene.com/ }\end{array}$ \\
\hline BLItzPro 1.2 software & FortéBio (Sartorius) & https://www.sartorius.com/ \\
\hline Prism 8.4.3 software & GraphPad & $\begin{array}{c}\text { https: } \\
\text { //www.graphpad.com/ }\end{array}$ \\
\hline
\end{tabular}

\subsubsection{Western Blotting}

Western blotting was performed as previously described [27] to check mTOR expression and purification steps. After SDS-PAGE, the protein bands were transferred onto PVDF membrane (Millipore (Merck), Darmstadt, Germany) using Trans-Blot Transfer System (Bio-Rad, Hercules, CA, USA). Then, the membrane was blocked for $1 \mathrm{~h}$ at RT with $4 \% w / v$ skimmed milk 1xTBS-T, and after wash, it was incubated for $1 \mathrm{~h}$ at RT with primary antibody against mTOR, followed by the goat anti-rabbit secondary antibody incubation at RT or $2 \mathrm{~h}$. Finally, the images of membranes were collected using the WSE-6100 LuminoGraph I (ATTO, Amherst, NY, USA).

\subsubsection{Preparation of RHEB and Truncated mTOR Fragments}

a Plasmid construction

The proteins were prepared by the pET15b expression vector and BL21(DE3) E. coli (Nippon Gene, Toyama, Japan) $[15,28]$. Briefly, RHEB (507 bp), $\Delta$ N-FAT-M (858 bp), $\Delta N$ (294 bp), and $\triangle$ ATP (459 bp) genes were cloned into pET15b expression vector using In-fusion cloning kit. The constructed plasmids were transformed into DH5 $\alpha$ E. coli (Nippon Gene, Toyama, Japan) and spread over Luria-Bertani (LB) agar medium (SigmaAldrich, St. Louis, MO, USA) plates containing $0.1 \mathrm{mM}$ ampicillin. Colony PCR and gene sequencing were performed to confirm the gene constructs and the plasmids were purified using NucleoSpin ${ }^{\circledR}$ Plasmid EasyPure kit (Macherey-Nagel, Duren, Germany) according to the manufacturer's protocol. Then, the purified plasmids were transformed into BL21(DE3) E. coli (Nippon Gene, Toyama, Japan) and incubated at $37^{\circ} \mathrm{C}$ for $6 \mathrm{~h}$ in LB media containing $0.1 \mathrm{mM}$ ampicillin. Protein expression was induced by $1 \mathrm{mM}$ IPTG and further incubated overnight at $37^{\circ} \mathrm{C}$. SDS-PAGE showed that RHEB and $\triangle \mathrm{N}$ were expressed as a soluble protein, while $\triangle \mathrm{N}$-FAT-M and $\triangle \mathrm{ATP}$ were expressed as inclusion bodies.

\section{b. Protein expression}

At day 1, 5 liters terrific broth (TB) medium (Sigma-Aldrich, St. Louis, MO, USA) was prepared containing $0.8 \%(v / v)$ glycerol and autoclaved. Parallelly, $5 \mathrm{~mL}$ starter cultures were prepared as described above without IPTG and incubated overnight at $37^{\circ} \mathrm{C}$. At day 2, $0.1 \mathrm{mM}$ ampicillin and $1 \%(v / v)$ antifoam silicon-type (SI) solution were added to the $\mathrm{TB}$ media and $\mathrm{OD}_{600}$ was checked as a reference. The starter cultures were added to the TB flasks and incubated at $37^{\circ} \mathrm{C}$ with shaking $(110 \mathrm{rpm})$. The $\mathrm{OD}_{600}$ values were measured hourly until they reached values $\geq 1.0$, then $1 \mathrm{mM}$ IPTG was added, and the culture was 
further incubated overnight. At day 3 , cultures were centrifuged at $8000 \mathrm{rpm}\left(15 \mathrm{~min}, 4^{\circ} \mathrm{C}\right)$, and the cell pastes were stored at $-80^{\circ} \mathrm{C}$.

c. Protein purification

Cell pastes ( $5 \mathrm{~g}$ for insoluble proteins or $10 \mathrm{~g}$ for soluble proteins) were resuspended in $100 \mathrm{~mL}$ lysis buffer (50 mM Tris-HCl, pH 8.0, $100 \mathrm{mM} \mathrm{NaCl}, 1 \mathrm{mM}$ EDTA, $0.04 \mathrm{mg} / \mathrm{mL}$ lysozyme, $0.16 \mathrm{mg} / \mathrm{mL}$ DNase I) supplemented with protease inhibitor cocktail tablets (cOmplete ${ }^{\mathrm{TM}}$, Roche (Merck), Darmstadt, Germany) and the suspensions were disrupted using a sonicator (Branson sonifier 250, Emerson Electric, St. Louis, MO, USA) on ice (5.0 W, 30-40\% cycle/s, $5 \mathrm{~min})$. For soluble proteins, cell lysate was ultra-centrifuged at $40,000 \mathrm{rpm}$ for $1 \mathrm{~h}$ at $4{ }^{\circ} \mathrm{C}$ and the pellet was discarded. Then, the supernatant was applied to the HisTrapTM HP Ni-NTA column purified by ÄKTAprime plus fast protein liquid chromatography (FPLC) (GE Healthcare, Chicago, IL, USA) using binding buffer (50 mM Tris- $\mathrm{HCl}, \mathrm{pH} 8.0,10 \%(v / v)$ glycerol) and eluted by a gradient $(0-100 \%)$ of elusion buffer (50 mM Tris- $\mathrm{HCl}, 10 \%(v / v)$ glycerol, $1 \mathrm{M}$ imidazole, $\mathrm{pH}$ 8.0). The peak fractions were checked by SDS-PAGE, pooled, and loaded onto $3 \mathrm{kDa}$ MW-CO Amicon Ultra ${ }^{\circledR}$ (Millipore (Merck), Darmstadt, Germany) and washed with the binding buffer. Finally, proteins were concentrated and stored at $-80^{\circ} \mathrm{C}$.

The recombinant $\Delta$ N-FAT-M and $\triangle$ ATP have been expressed as inclusion bodies, so the purification process involved a refolding step. After cell disruption, the lysate was centrifuged at $8000 \mathrm{rpm}$ for $5 \mathrm{~min}$ at $4{ }^{\circ} \mathrm{C}$. Then, the pellet was washed 3 times by washing buffer ( $50 \mathrm{mM}$ Tris- $\mathrm{HCl}, 100 \mathrm{mM} \mathrm{NaCl}, 1 \mathrm{mM}$ EDTA, $4 \mathrm{M}$ urea, $\mathrm{pH}$ 8.0) followed by 3 times washing by the same buffer without urea. The pellet was then resuspended in $20 \mathrm{~mL}$ solubilization buffer $\left(50 \mathrm{mM}\right.$ Tris- $\mathrm{HCl}, 8 \mathrm{M}$ urea, $100 \mathrm{mM} \mathrm{NaH} \mathrm{PO}_{4}, 10 \mathrm{mM}$ 2-mercaptoethanol, $\mathrm{pH} 8.0)$ on a rotator for $2 \mathrm{~h}$ at RT. After centrifugation $(40,000 \mathrm{rpm}$, $\left.1 \mathrm{~h}, 4^{\circ} \mathrm{C}\right)$, the supernatant was diluted by the binding buffer $(50 \mathrm{mM}$ Tris- $\mathrm{HCl}, 8 \mathrm{M}$ urea, $100 \mathrm{mM} \mathrm{NaH}_{2} \mathrm{PO}_{4}, 5 \mathrm{mM}$ 2-mercaptoethanol, $\mathrm{pH}$ 8.0) and loaded onto HisTrapTM FF crude Ni-NTA column (GE Healthcare, Chicago, IL, USA). After binding, the protein was eluted by a gradient $(0-100 \%)$ of elusion buffer $\left(50 \mathrm{mM}\right.$ Tris- $\mathrm{HCl}, 8 \mathrm{M}$ urea, $100 \mathrm{mM} \mathrm{NaH} \mathrm{PO}_{4}$, $5 \mathrm{mM}$ 2-mercaptoethanol, $1 \mathrm{M}$ imidazole, $\mathrm{pH}$ 8.0). The peak fractions were checked by SDS-PAGE, pooled, and dropped into refolding buffer $(50 \mathrm{mM}$ Tris- $\mathrm{HCl}, 40 \mathrm{mM} \mathrm{NaCl}$, $1 \mathrm{mM}$ EDTA, $1 \mathrm{M} \mathrm{L}$-arginine, and 10\% ( $v / v)$ glycerol, $\mathrm{pH}$ 8.0). The refolded sample was then washed over a $3 \mathrm{kDa}$ MW-CO filter (Millipore (Merck), Darmstadt, Germany) by the gel filtration buffer. After that, proteins were subjected to gel filtration using Superdex-200 column and running buffer of $50 \mathrm{mM}$ Tris- $\mathrm{HCl}, \mathrm{pH} 8.0,150 \mathrm{mM} \mathrm{NaCl}, 10 \%(v / v)$ glycerol. After checking the purity on SDS-PAGE, the purified fractions were collected, concentrated by $3 \mathrm{kDa}$ MW-CO Amicon Ultra ${ }^{\circledR}$ (Millipore (Merck), Darmstadt, Germany), and the aliquots were stored at $-80^{\circ} \mathrm{C}$.

\subsubsection{His-Tag Cleavage from RHEB}

The RHEB aliquot containing $1 \mathrm{mg}$ of RHEB was diluted 20 times in 1X PBS, and then mixed with 10 units of thrombin and incubated for $16 \mathrm{~h}$ at RT on a rotator to cleave the 6-His tag at the thrombin-cleavage site. Then, the solution was passed over the His SpinTrapTM column (GE Healthcare, Chicago, IL, USA) to remove 6-His tag which was eluted by 100 , 200 , and $500 \mathrm{mM}$ imidazole in 1X PBS, respectively. To remove thrombin, the fractions were loaded onto HiTrapTM Benzamidine FF column (GE Healthcare, Chicago, IL, USA) equilibrated by binding buffer $\mathrm{C}(50 \mathrm{mM}$ Tris- $\mathrm{HCl}, 100 \mathrm{mM} \mathrm{NaCl}, \mathrm{pH} 8.0)$, and thrombinfree RHEB was gradually (0-100\%) eluted by the elution buffer B ( $50 \mathrm{mM}$ Tris- $\mathrm{HCl}, 500 \mathrm{mM}$ $\mathrm{NaCl}, \mathrm{pH}$ 8.0).

\subsubsection{RHEB Charging with GTP $\gamma \mathrm{S}$}

We followed the RHEB charging protocol as previously described, with some modifications [11]. RHEB was incubated with 20-fold molar excess of GTP $\gamma \mathrm{S}$ (Millipore (Merck), Darmstadt, Germany) in the presence of $10 \mathrm{mM}$ EDTA for $20 \mathrm{~min}$ at RT. Finally, the reaction 
was stopped by the addition of $20 \mathrm{mM}$ of $\mathrm{MgCl}_{2}$. The yield was then passed over a PD SpinTrap G-25 column (GE Healthcare, Chicago, IL, USA) to remove excess GTP $\gamma$ S.

\subsubsection{AlphaLISA Assay for RHEB-mTOR Protein-Protein Interaction (PPI)}

A PPI assay based on energy transfer via donor/acceptor system using AlphaLISA ${ }^{\circledR}$ assay (PerkinElmer, Waltham, MA, USA) was used [29]. Briefly, $100 \mu \mathrm{g} / \mathrm{mL}$ anti-IgG donor beads were incubated with excess anti-mTOR antibody for $1 \mathrm{~h}$ at $\mathrm{RT}$ followed by washing by $1 \times$ dilution buffer supplemented with the beads to remove unbound anti-mTOR antibodies. Then, a final concentration of $10 \mathrm{nM}$ of purified mTOR was added to the donor beads solution and was further incubated for $1 \mathrm{~h}$ at RT. After the washes, the donor beadsmTOR complex was aliquoted $(4 \mu \mathrm{L})$ in a 384-well OptiPlateTM (PerkinElmer, Waltham, MA, USA). Then, $6 \times$ His-RHEB or $6 x$ His-RHEB-GTP $\gamma$ S was titrated $(3 \mu \mathrm{L}$ of $0.1-100 \mu \mathrm{M})$ into the donor beads-mTOR mix and shaken gently. Finally, $3 \mu \mathrm{L}$ of $200 \mu \mathrm{g} / \mathrm{mL}$ anti6 His acceptor beads were added to the mixture, the plate was top-sealed, covered, and incubated for more than $1 \mathrm{~h}$ at RT in dark. The same steps were performed to obtain baseline but without the addition of RHEB. The alpha signals were then measured by EnSpire $^{\mathrm{TM}}$ plate reader (PerkinElmer, Waltham, MA, USA). The data was normalized by subtracting the baseline values and the $\mathrm{K}_{\mathrm{D}}$ values were calculated by the Prism software by using the specific binding model with the equation: $Y=B_{\max }(X /(K d+X))$, where $Y$, specific binding (measurement signal unit); $\mathrm{B}_{\max }$, maximum binding (measurement signal unit); $X$, concentration of the analyte; $\mathrm{Kd}$, the binding affinity.

\subsubsection{Preparation of Plasmids for In-Cell Protein-Protein Interaction (NanoBiT Assay)}

RHEB (507 bp) gene was cloned into LgBiT vector, and $\triangle \mathrm{N}-F A T-M(858 \mathrm{bp}), \Delta \mathrm{N}-\mathrm{M}$ (495 bp), $\Delta \mathrm{N}$ (294 bp), $\Delta \mathrm{M}(171 \mathrm{bp})$, and $\triangle \mathrm{ATP}(459 \mathrm{bp}$ ) genes were cloned into SmBiT vector using In-fusion cloning kit. The constructed plasmids were transformed into Dh5 $\alpha$ E. coli and the cultures were spread over LB agar plates containing $0.1 \mathrm{mM}$ ampicillin. Colony PCR and gene sequencing were performed to confirm the gene constructs, and the plasmids were purified using NucleoSpin ${ }^{\circledR}$ Plasmid EasyPure kit (Macherey-Nagel, Duren, Germany) according to the manufacturer's protocol.

\subsubsection{In-Cell NanoBiT Assay}

The NanoLuc Binary Technology (NanoBiT, Promega, Madison, WI, USA) based on split luciferase subunits can be used for the intracellular detection of PPI [21]. Briefly, $10^{4}$ HEK293 cells were seeded in DMEM (10\% fetal bovine serum (FBS); $1 \%$ penicillin/streptomycin $(\mathrm{P} / \mathrm{S})$ ) in B\&W Isoplate-96 tissue-culture (TC) treated plates (PerkinElmer, Waltham, MA, USA) and incubated overnight $\left(5 \% \mathrm{CO}_{2} ; 37^{\circ} \mathrm{C}\right)$. Then, the RHEB-LgBiT vector with the different $\mathrm{SmBiT}$ variants ( $50 \mathrm{ng} /$ well each) were co-transfected into the cells using FuGENE HD at a ratio of 3:1 $(v / w)$ and incubated for $48 \mathrm{~h}\left(5 \% \mathrm{CO}_{2} ; 37^{\circ} \mathrm{C}\right)$ for protein expression. Then, the culture medium was replaced by Opti-MEM reduced serum medium (Gibco, ThermoFisher, Waltham, MA, USA), and $20 \mu \mathrm{L}$ of 20-fold diluted furimazine (Nano-Glo, Promega, Madison, WI, USA) was injected to initiate the luciferase reaction; the luminescence signal was measured using EnSpire plate reader (PerkinElmer, Waltham, MA, USA).

\subsubsection{BLItz Measurements of RHEB Interactions with mTOR Truncates}

We used the BLItz instrument (FortéBio, Fremont, CA, USA) to measure the binding kinetics of RHEB with the truncated mTOR fragment. At first, Ni-NTA biosensors (FortéBio, Fremont, CA, USA) were hydrated for $2 \mathrm{~h}$ in the kinetics buffer (10 mM HEPES, $\mathrm{pH} 7.4,100 \mathrm{mM} \mathrm{NaCl}, 0.02 \%(v / v)$ Tween-20, and $5 \mathrm{mg} / \mathrm{mL}$ bovine serum albumin). In all measurements, the his-tagged truncated mTOR fragments $(1 \mu \mathrm{M})$ were used as ligands to be immobilized onto the sensors, while tagless RHEB was used as analyte. In case of $\Delta \mathrm{N}$-FAT-M or $\Delta \mathrm{N}$, the measurement cycle composed of $30 \mathrm{~s}$ initial baseline (buffer), $120 \mathrm{~s}$ ligand loading, $120 \mathrm{~s}$ baseline (buffer), $180 \mathrm{~s}$ analyte association, and $300 \mathrm{~s}$ dissociation phases (buffer), while for $\triangle \mathrm{ATP}$, the cycle was shorter and divided into $30 \mathrm{~s}, 120 \mathrm{~s}, 60 \mathrm{~s}$, 
120 s, and 240 s, respectively. A reference cycle was applied for each sensor by introducing analyte only in the association phase to exclude nonspecific binding possibilities. RHEB concentrations were 0.5 and $1 \mu \mathrm{M} \Delta \mathrm{N}-\mathrm{FAT}-\mathrm{M}$ or $\Delta \mathrm{N}$, and 0.05 and $0.1 \mu \mathrm{M}$ for $\Delta \mathrm{ATP}$. All the experiments were performed at a shaking speed of $2000 \mathrm{rpm}$ at $25^{\circ} \mathrm{C}$. Finally, the binding curves were fitted using 1:1 binding kinetics and analyzed by the BLItz Pro 1.2 software (FortéBio, Fremont, CA, USA) with the equations:

Association:

$$
\mathrm{Y}=\mathrm{Y}_{0}+\mathrm{R}_{\mathrm{eq}}\left(1-\mathrm{e}^{-\mathrm{Kobs} \times \mathrm{t}}\right)
$$

Dissociation:

$$
\mathrm{Y}=\mathrm{Y}_{\mathrm{e}}+\mathrm{Y}_{\Delta} \mathrm{e}^{-\mathrm{Kd} \times \mathrm{t}}
$$

$\mathrm{Y}$, BLI signal in nm; $\mathrm{Y}_{0}$, the initial binding level; $\mathrm{Y}_{\mathrm{e}}$, the fitted value of the exponential decay curve; $\mathrm{Y}_{\Delta}$, the nm shift difference between the first data point of the fitted dissociation curve and $\mathrm{Y}_{\mathrm{e}} ; \mathrm{R}_{\mathrm{eq}}, \mathrm{R}$ equilibrium; $\mathrm{K}_{\mathrm{obs}}$, the observed rate constant; $\mathrm{K}_{\mathrm{d}}$, the dissociation rate constant; $t$, time in seconds.

\subsubsection{Data Analysis}

Statistical significance and number of samples are noted in the figure legends where appropriate. Data are expressed as mean \pm SD. Ordinary one-way ANOVA was used as indicated; $^{* * * *}$ for $p<0.0001$ and ns for $p>0.05$. Statistical analyses were performed using GraphPad Prism software, v.8.4.3 (GraphPad, San Diego, CA, USA).

\section{Conclusions}

Although the previous studies showed that RHEB-GTP activated mTORC1 by several mechanisms, they did not reveal the binding kinetics $[4,8,10]$. Here, we studied the binding details of RHEB to whole mTOR and the truncated mTOR fragments $[4,10]$. In the assays, we used the in-cell and in vitro assays to facilitate the measurements $[15,16]$. RHEB bound to whole mTOR with 5 times weaker affinity in the presence of GTP than in its absence. On the other hand, the binding study of the truncated mTOR fragments involved in the reconstituted allosteric binding site suggested the cooperative binding mode of N-heat, M-heat, and FAT domains for RHEB. In addition, we observed that RHEB bound to the truncated ATP binding site in-cell and in vitro. The results show that the binding of RHEB to mTOR involves multiple binding sites with a variety of biding affinities, suggesting that RHEB regulates the kinase activity of mTOR through multiple mechanisms.

Supplementary Materials: The following are available online at https:/ / www.mdpi.com/article/10 $.3390 /$ ijms22168766/s1.

Author Contributions: H.M. and R.S. conceived the whole project. Y.I. managed the research group. R.S. designed and conducted the experimental works. R.S. and H.M. wrote the manuscript. All authors checked and approved the experimental results and the manuscript. All authors have read and agreed to the published version of the manuscript.

Funding: R.S. was supported by the Junior Research Associate (JRA) Program in RIKEN. H.M. was partly supported by the Incentive Research Program in RIKEN (FY2018-2019, FY2019-2020) and JSPS KAKENHI Grant Number JP20K06516.

Institutional Review Board Statement: Not applicable.

Informed Consent Statement: Not applicable.

Data Availability Statement: Not applicable.

Acknowledgments: We are grateful to the Support Unit for Bio-material Analysis, RIKEN CBS Research Resources Center (RRC) for DNA sequencing.

Conflicts of Interest: The authors declare no competing interests. 


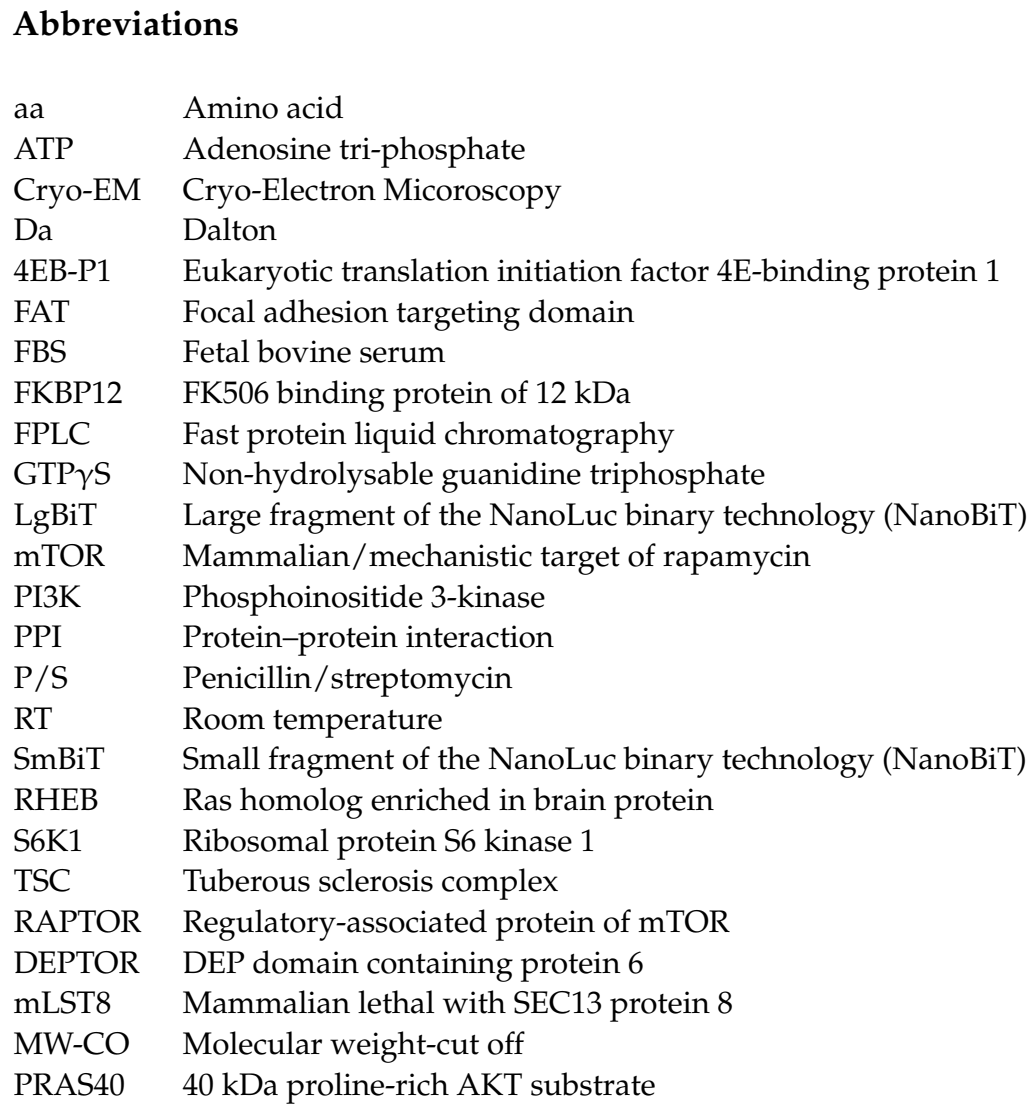

\section{References}

1. Liu, G.Y.; Sabatini, D.M. mTOR at the Nexus of Nutrition, Growth, Ageing and Disease. Nat. Rev. Mol. Cell Biol. 2020, 21, 183-203. [CrossRef]

2. Mossmann, D.; Park, S.; Hall, M.N. mTOR Signalling and Cellular Metabolism are Mutual Determinants in Cancer. Nat. Rev. Cancer 2018, 18, 744-757. [CrossRef]

3. Saxton, R.A.; Sabatini, D.M. mTOR Signaling in Growth, Metabolism, and Disease. Cell 2017, 169, 361-371. [CrossRef]

4. Yang, H.; Jiang, X.; Li, B.; Yang, H.J.; Miller, M.; Yang, A.; Dhar, A.; Pavletich, N.P. Mechanisms of mTORC1 activation by RHEB and inhibition by PRAS40. Nature 2017, 552, 368-373. [CrossRef] [PubMed]

5. Shen, K.; Sabatini, D.M. Ragulator and SLC38A9 activate the Rag GTPases through noncanonical GEF mechanisms. Proc. Natl. Acad. Sci. USA 2018, 115, 9545-9550. [CrossRef]

6. Saxton, R.A.; Chantranupong, L.; Knockenhauer, K.E.; Schwartz, T.U.; Sabatini, D.M. Mechanism of arginine sensing by CASTOR1 upstream of mTORC1. Nature 2016, 536, 229-233. [CrossRef] [PubMed]

7. Heard, J.J.; Fong, V.; Bathaie, S.Z.; Tamanoi, F. Recent progress in the study of the Rheb family GTPases. Cell Signal 2014, 26, 1950-1957. [CrossRef] [PubMed]

8. Bai, X.; Ma, D.; Liu, A.; Shen, X.; Wang, Q.J.; Liu, Y.; Jiang, Y. RHEB Activates mTOR by Antagonizing its Endogenous Inhibitor, FKBP38. Science 2007, 318, 977-980. [CrossRef] [PubMed]

9. Long, X.; Ortiz-Vega, S.; Lin, Y.; Avruch, J. Rheb binding to mammalian target of rapamycin (mTOR) is regulated by amino acid sufficiency. J. Biol. Chem. 2005, 280, 23433-23436. [CrossRef]

10. Long, X.; Lin, Y.; Ortiz-Vega, S.; Yonezawa, K.; Avruch, J. Rheb binds and regulates the mTOR kinase. Curr. Biol. 2005, 15, 702-713. [CrossRef]

11. Mahoney, S.J.; Narayan, S.; Molz, L.; Berstler, L.A.; Kang, S.A.; Vlasuk, G.P.; Saiah, E. A Small Molecule Inhibitor of Rheb Selectively Targets mTORC1 Signaling. Nat. Commun. 2018, 9, 1-12. [CrossRef]

12. Castro, A.F.; Rebhun, J.F.; Clark, G.J.; Quilliam, L.A. RHEB Binds Tuberous Sclerosis Complex 2 (TSC2) and Promotes S6 Kinase Activation in a Rapamycin- and Farnesylation-Dependent Manner. J. Biol. Chem. 2003, 278, 32493-32496. [CrossRef]

13. Rogala, K.B.; Gu, X.; Kedir, J.F.; Abu-Remaileh, M.; Bianchi, L.F.; Bottino, A.M.S.; Dueholm, R.; Niehaus, A.; Overwijn, D.; Fils, A.P.; et al. Structural basis for the docking of mTORC1 on the lysosomal surface. Science 2019, 366, 468-475. [CrossRef]

14. Anandapadamanaban, M.; Masson, G.R.; Perisic, O.; Berndt, A.; Kaufman, J.; Johnson, C.M.; Santhanam, B.; Rogala, K.B.; Sabatini, D.M.; Williams, R.L. Architecture of Human Rag GTPase Heterodimers and their Complex with mTORC1. Science 2019, 366 , 203-210. [CrossRef]

15. Ning, B.; Ren, X.; Hagiwara, K.; Takeoka, S.; Ito, Y.; Miyatake, H. Development of a Non-IgG PD-1/PD-L1 Inhibitor by in Silico Mutagenesis and an In-Cell Protein-Protein Interaction Assay. ACS Chem. Biol. 2021, 16, 316-323. [CrossRef] [PubMed] 
16. Shams, R.; Matsukawa, A.; Ochi, Y.; Ito, Y.; Miyatake, H. In Silico and In Cell Hybrid Selection of Nonrapalog Ligands to Allosterically Inhibit the Kinase Activity of mTORC1. J. Med. Chem. 2021. [CrossRef]

17. Unni, N.; Arteaga, C.L. Is Dual mTORC1 and mTORC2 Therapeutic Blockade Clinically Feasible in Cancer? JAMA Oncol. 2019, 5, 1564-1565. [CrossRef]

18. Srivastava, R.K.; Li, C.; Khan, J.; Banerjee, N.S.; Chow, L.T.; Athar, M. Combined mTORC1/mTORC2 inhibition blocks growth and induces catastrophic macropinocytosis in cancer cells. Proc. Natl. Acad. Sci. USA 2019, 116, 24583-24592. [CrossRef] [PubMed]

19. Hua, H.; Kong, Q.; Zhang, H.; Wang, J.; Luo, T.; Jiang, Y. Targeting mTOR for cancer therapy. J. Hematol. Oncol. 2019, 12, 1-19. [CrossRef] [PubMed]

20. Kang, S.A.; O’Neill, D.J.; Machl, A.W.; Lumpkin, C.J.; Galda, S.N.; Sengupta, S.; Mahoney, S.J.; Howell, J.J.; Molz, L.; Hahm, S.; et al. Discovery of Small-Molecule Selective mTORC1 Inhibitors via Direct Inhibition of Glucose Transporters. Cell Chem. Biol. 2019, 26, 1203-1213. [CrossRef]

21. Dixon, A.S.; Schwinn, M.K.; Hall, M.P.; Zimmerman, K.; Otto, P.; Lubben, T.H.; Butler, B.L.; Binkowski, B.F.; Machleidt, T.; Kirkland, T.A.; et al. NanoLuc Complementation Reporter Optimized for Accurate Measurement of Protein Interactions in Cells. ACS Chem. Biol. 2016, 11, 400-408. [CrossRef] [PubMed]

22. Gehrmann, T.; Heilmeyer, L.M., Jr. Phosphatidylinositol 4-kinases. Eur. J. Biochem. 1998, 253, 357-370. [CrossRef]

23. Sato, T.; Nakashima, A.; Guo, L.; Tamanoi, F. Specific activation of mTORC1 by Rheb G-protein in vitro involves enhanced recruitment of its substrate protein. J. Biol. Chem. 2009, 284, 12783-12791. [CrossRef]

24. Yang, H.; Rudge, D.G.; Koos, J.D.; Vaidialingam, B.; Yang, H.J.; Pavletich, N.P. mTOR kinase structure, mechanism and regulation. Nature 2013, 497, 217-223. [CrossRef]

25. Deplus, R.; Delatte, B.; Schwinn, M.K.; Defrance, M.; Mendez, J.; Murphy, N.; Dawson, M.A.; Volkmar, M.; Putmans, P.; Calonne, E.; et al. TET2 and TET3 regulate GlcNAcylation and H3K4 methylation through OGT and SET1/COMPASS. EMBO J. 2013, 32, 645-655. [CrossRef]

26. Ohana, R.F.; Hurst, R.; Vidugiriene, J.; Slater, M.R.; Wood, K.V.; Urh, M. HaloTag-based purification of functional human kinases from mammalian cells. Protein Expr. Purif. 2011, 76, 154-164. [CrossRef]

27. Nguyen, T.L.; Nokin, M.J.; Egorov, M.; Tome, M.; Bodineau, C.; Di Primo, C.; Minder, L.; Wdzieczak-Bakala, J.; Garcia-Alvarez, M.C.; Bignon, J.; et al. mTOR Inhibition via Displacement of Phosphatidic Acid Induces Enhanced Cytotoxicity Specifically in Cancer Cells. Cancer Res. 2018, 78, 5384-5397. [CrossRef]

28. Dharmatti, R.; Miyatake, H.; Zhang, C.; Ren, X.; Yumoto, A.; Kiga, D.; Yamamura, M.; Ito, Y. Escherichia coli expression, purification, and refolding of human folate receptor alpha (hFRalpha) and beta (hFRbeta). Protein Expr. Purif. 2018, 149, 17-22. [CrossRef] [PubMed]

29. Yasgar, A.; Jadhav, A.; Simeonov, A.; Coussens, N.P. AlphaScreen-Based Assays: Ultra-High-Throughput Screening for SmallMolecule Inhibitors of Challenging Enzymes and Protein-Protein Interactions. Methods Mol. Biol. 2016, 1439, 77-98. [CrossRef] [PubMed] 